\title{
Fecal Microbiota Transplantation as a Novel Method of Multidrug-Resistant Infections Treatment in Allogeneic Hematopoietic Stem Cell Transplantation Recipients
}

\author{
Maxim Kucher ${ }^{1 *}$, Oleg Goloshchapov ${ }^{1}$, Maria Suvorova ${ }^{2}$, Ruslana Klementeva ${ }^{1}$, Aleksandr Shcherbakov ${ }^{1}$, Alexandr Shvetcov $^{1}$, \\ Ilya Kazantsev ${ }^{1}$, Ivan Moiseev ${ }^{1}$, Ludmila Zubarovskaya ${ }^{1}$ and Boris Afanasyev ${ }^{1}$
}

${ }^{1}$ R. Gorbacheva Memorial Institute for Children Oncology, Hematology and Transplantation, Pavlov First Saint-Petersburg State Medical University, St. Petersburg, Russia

${ }^{2}$ Scientific research laboratory Explana, Saint-Petersburg, Russia

*Corresponding author: Dr. Maxim Kucher, Head, Department of Clinical Nutrition, R. Gorbacheva Memorial Institute for Children Oncology, Hematology and Transplantation, Pavlov First Saint-Petersburg State Medical University, St. Petersburg, Russia, Tel: 8 (812) 338 6260; E-mail: doctorkucher@yandex.ru

Received: 26 Oct, 2017 | Accepted: 12 Jan, 2018 | Published: 18 Jan, 2018

Citation: Kucher M, Goloshchapov O, Suvorova M, Klementeva R, Shcherbakov A, et al. (2018) Fecal Microbiota Transplantation as a Novel Method of Multidrug-Resistant Infections Treatment in Allogeneic Hematopoietic Stem Cell Transplantation Recipients. Transplant Res J 3(1): dx.doi.org/10.16966/2473-1730.117

Copyright: (C) 2018 Kucher M, et al. This is an open-access article distributed under the terms of the Creative Commons Attribution License, which permits unrestricted use, distribution, and reproduction in any medium, provided the original author and source are credited.

\begin{abstract}
This short communication accompanies our presentation at the 43rd Annual meeting of the European Society for Blood and Marrow Transplantation held in Marseille, France, 26-29 March 2017. Also it briefly summaries some recent advances in fecal microbiota transplantation (FMT) for treatment of infections in allogeneic hematopoietic stem cell transplantation (allo-HSCT) recipients. AlloHSCT is an effective method of treatment for some hematological, malignant and hereditary conditions in adults and children. However, allo-HSCT is also associated with several potentially life-threatening complications. Among the most common causes for transplantassociated mortality are antibiotic-resistant bloodstream infections and immune complications, such as acute and chronic graft-versushost disease (GvHD). In most cases, gastrointestinal tract (GIT) is primarily damaged post-HSCT due to enhanced inflammation, GvHD, and intestinal infections causing serious diarrhea, in particular pseudomembranous colitis associated with Clostridium difficile, which often develops in patients receiving massive antibiotic therapy. Normal intestinal microbiota elimination is itself an important risk factor for sepsis, GIT GvHD, pseudo-membranous colitis and antibiotic-associated diarrhea post-HSCT. FMT from healthy donors allows restoring the physiological variability and functional activity of intestinal microbiota leading to pathogens eradication in patients with GIT infections.
\end{abstract}

Keywords: Fecal microbiota transplantation; Hematopoietic stem cell transplantation; Antibiotic resistance

Abbreviations: Allogeneic Hematopoietic Stem Cell Transplantation allo-HSCT; Fecal Microbiota Transplantation - FMT; Gastrointestinal Tract - GIT; Graft versus Host Disease - GvHD

\section{Introduction}

Allogeneic hematopoietic stem cell transplantation (alloHSCT) is an effective treatment method for some malignant and non-malignant (autoimmune and hereditary) conditions in children and adults. The hematopoietic stem cell infusion is preceded by conditioning regimen consisting of cytostatics and/or irradiation, which allows restoring donor-derived normal hemopoiesis in cases of bone marrow damage or malfunctioning [1].

The HSCT efficacy is limited by several main factors. If HSCT is performed in a patient with malignant condition there is usually a problem of primary or secondary chemotherapy resistance leading to high risk of post-HSCT relapse. Also, there is significant mortality due to septic complications caused by nosocomial multi-drug resistant bacteria strains with prevalence of Clostridium difficile, Klebsiella pneumonia, Pseudomonas aeruginosa and Vancomycin-resistant Enterococci. At last, recipient tissues and organs may be affected by donor lymphocytes-mediated immune complications, such as acute and chronic graft-versus-host disease (GvHD) [2].

Gastrointestinal tract (GIT) is a primary target for most allo-HSCT associated harmful factors, which often lead to immune-mediated inflammation and serious diarrhea due to GvHD and intestinal infections, in particular in patients with post antibacterial therapy Clostridium difficile-associated pseudomembranous colitis. Allo-HSCT negatively influences patient's normal microbiota due to a number of specific factors, e.g. low-microbial diet often leading to malnutrition, intestinal decontamination associated with obligate microorganisms eradication, and the use of cytotoxic drugs and/or radiation directly damaging GIT epithelial cells. Together with antibiotic therapy these factors produce severe bacterial microbiota alterations with development of pathogenic multidrugresistant flora, which presumably increases the risk of acute GvHD [3,4]. Post allo-HSCT mortality is known to be 
significantly higher in patients with skewed biovariability of normal microbiota [5].

While there is a growing body of data on FMT effectiveness and tolerability, the indications for this procedure are presently being expanded. It is currently used in resistant Clostridium difficile infection [6], inflammatory bowel disease, irritable bowel syndrome [7], rheumatoid arthritis, type 2 diabetes, autism, chronic fatigue syndrome, multiple sclerosis and Parkinson's disease [8]. The main known mechanisms of FMT action include competition for nutrients, direct pathogen growth inhibition and modulation of host immune system by interaction with normal microbiota.

Despite some encouraging results of FMT-assisted treatment, it is still rarely used in severely immunocompromised patients, e.g. allo-HSCT recipients, since there is a risk of microorganism's translocation to systemic circulation via damaged intestinal wall and generalized infection development. There are only some reports on FMT performed in this population for sepsis with multiple organ dysfunction syndrome caused by vancomycinresistant pathogens [9]. Innes A.J. et al. [10] described a successful case of pre allo-HSCT FMT in a patient with acute leukemia aimed to eradicate a persistent infection developing after post-cytostatic sepsis antibacterial treatment. Several FMTs were performed in pediatric allo-HSCT recipients with Clostridium difficile infection [11].

An important point is that transplanted microorganisms may substitute multidrug-resistant flora as its natural antagonists potentially providing more durable control over infections compared to standard antibiotics.

\section{Materials and Methods}

Three R. Gorbacheva Memorial Institute for Children Oncology, Hematology and Transplantation patients (a 3 year old boy with beta-thalassemia, 10 year old girl with acute lymphoblastic leukemia and 28 year old man with acute myeloid leukemia) with toxin-B positive pseudomembranous colitis accompanying acute or chronic GVHD after allo-HSCT from haploidentical donor were enrolled. All these three patients received FMT in 2016.

After the indications for FMT were confirmed, an informed consent for the procedure was signed by a patient or his/ her caregiver and donor. All microbiota donors were related (patient's mother, father or sibling). Before FMT both donor and patient underwent routine investigation including $\mathrm{CBC}$, serum biochemistry, microbiota cultures and multiplex realtime polymerase chain reaction (e.g. Colonoflor-16, a Lab, Russia) for most common intestinal microorganisms. For patients also the ELISA fecal calprotectin level was assessed, drug-resistant pathogens were identified and intestinal bioptate morphology was evaluated. In order to prepare for FMT the patient received probiotic (inulin) 72 hours prior to procedure discontinued all antibiotics 24 hours before. Also, antiemetics (5HT3-agonist), prokinetics and proton pump inhibitor were added. The standard transplant preparation procedure was used [12]. Donor's microbiota was delivered in two consecutive steps under total intravenous anesthesia to duodenum (with esophagogastroduodenoscopy) and caecum (with colonoscopy).

\section{Results and Discussion}

All patients included in the study obtained complete clinical response with vomiting, nausea, abdominal pain, diarrhea, fever and anorexia symptoms regression 14-28 days post FMT (Table 1). No adverse effects were observed. In 10 days we have revealed significant quantitative and qualitative changes in patient's gut microbiota composition, which was shifting towards donor's one. In 35 days after FMT we identified similar changes in oropharynx and urogenital tract flora. The multidrug-resistant Klebsiella pneumoniae strains were substituted by drug-sensitive microorganisms leading to postallo-HSCT infection complications symptoms regression. Also, there were no clinical and morphological signs of intestinal GvHD.

FMT allows substitution of both GIT and other body areas (mouth, lungs, urinary routes, etc.) microbiota by less pathogenic species, which are more sensitive to antibiotics. This effect can be used to eradicate antibiotic-resistant pathogenic bacteria strains due to inter-microorganism natural competition and antagonism [13].

According to our data, diarrhea after allo-HSCT as a result of GIT dysfunction was observed in up to $79 \%$ of cases and may persist for several months leading gradually to cachexia and lethal infectious complications development. Thus, there is potentially a large population, in which FMT could be beneficial.

Hence, the general idea of using FMT in HSCT looks promising in many aspects: infectious complications prevention and treatment, malabsorption syndrome prophylaxis, and cachexia prevention, as well as steroid-resistant gut GVHD treatment [14].

Despite the encouraging treatment results, the FMT is rarely used in immunocompromised populations including allo-HSCT recipients. The reason is usually in relatively low performance status, dramatic structural changes of gastrointestinal mucosa, and concomitant infectious complications. Therefore, the routine use of FMT in patients after HSCT and in patients with secondary immunodeficiency may be associated with a threat of generalized infection caused by transplanted microbiota. However, it is worth noting that fecal transplantderived bacteria are less virulent and more sensitive to standard antibiotics. For example, in the clinical case no 2, the presence of $4 \times 10^{8} \mathrm{CFU} / \mathrm{ml}$ donors Proteus mirabilis (normal value is $\leq$ $10^{4} \mathrm{CFU} / \mathrm{ml}$ ) may have allowed to displace multidrug-resistant Klebsiella pneumoniae from other locations.

\section{Conclusion}

Post-HSCT infectious complications caused by standard 
Table 1: Patients' characteristics and FMT outcome

\begin{tabular}{|c|c|c|c|c|c|c|}
\hline Patient & $\begin{array}{c}\text { Number of } \\
\text { FMTs }\end{array}$ & $\begin{array}{c}\text { Diarrhea before } \\
\text { FMT, months }\end{array}$ & $\begin{array}{c}\text { Clostridium difficile } \\
\text { toxin B before FMT }\end{array}$ & $\begin{array}{c}\text { Clostridium difficile } \\
\text { toxin B after FMT }\end{array}$ & $\begin{array}{c}\text { Calprotectin before } \\
\text { FMT, mcg/g }\end{array}$ \\
\hline 1 & 2 & 6 & Positive & Negative & 290 \\
FMT, mcg/g \\
duration, weeks
\end{tabular}

antimicrobial therapy-resistant bacteria are often the cause of post-transplant sepsis-related mortality. The most common pathogens are Klebsiella pneumoniae, Pseudomonas aeruginosa, Clostridium difficile, and Acinetobacter baumannii. Prolonged antibiotic therapy seems to be a key factor for gut bacteria multidrug resistance development, which also may be a trigger for severe GvHD.

Initial clinical experience with effective and safe FMT treatment in heavily pretreated patients allows considering this method as an addition or alternative treatment method for infectious and immune post allo-HSCT complications. FMT causes intestinal microbiota composition changes able to help in multidrug-resistant gut pathogens (e.g. Clostridium difficile, Klebsiella pneumonia etc.) eradication. It allows reduction of antibiotic-associated diarrhea severity and replacement of resistant bacteria species in other body areas thus changing the patient's microbial profile to less virulent one.

Further FMT implementation in clinical practice requires a detailed study aimed at therapy initiation criteria optimization, selection of the most matched donors by means of multiplex DNA diagnostics or next-generation DNA sequencing of microbiota, and evaluation transplant long-term efficacy and safety.

\section{References}

1. Slavin S (2000) New strategies for bone marrow transplantation. Curr Opin Immunol 12: 542-551.

2. Afanasyev BV, Zubarovskaya LS, Moiseev IS (2015) Allogeneic hematopoietic stem cell transplantation in children: now, problems and prospects. Russian Journal of Children Hematology and Oncology 2: $28-42$.

3. Jenq RR, Ubeda C, Taur Y, Menezes CC, Khanin R, et al. (2012) Regulation of intestinal inflammation by microbiota following allogeneic bone marrow transplantation. J Exp Med 209: 903-911.
4. Shono Y, Docampo MD, Peled JU, Perobelli SM, Jenq RR (2015) Intestinal microbiota-related effects on graft-versus-host disease. Int J Hematol 101: 428-437.

5. Jenq RR, Taur $Y$, Devlin SM, Ponce DM, Goldberg JD, et al. (2015) Intestinal Blautia Is Associated with Reduced Death from Graft-versusHost Disease. Biol Blood Marrow Transplant 21: 1373-1383.

6. Cammarota G, lanitro G, Gasbarrini A (2014) Fecal microbiota transplantation for the treatment of Clostridium difficile infection: a systematic review. J Clin Gastroenterol 48: 693-702.

7. Rossen NG, MacDonald JK, de Vries EM, D'Haens GR, de Vos WM, et al. (2015) Faecal microbiota transplantation as novel therapy in gastroenterology: A systematic review. World J Gastroenterol 21: 53595371.

8. Evrensel A, Ceylan ME (2016) Fecal Microbiota Transplantation and Its Usage in Neuropsychiatric Disorders. Clin Psychopharmacol Neurosci 14: 231-237.

9. Wei Y, Yang J, Wang J, Yang Y, Huang J, et al. (2016) Successful treatment with fecal microbiota transplantation in patients with multiple organ dysfunction syndrome and diarrhea following severe sepsis. Crit Care 20: 332 .

10. Innes AJ, Mullish BH, Fernando F, Adams G, Marchesi JR, et al. (2017) Faecal microbiota transplant: a novel biological approach to extensively drug-resistant organism-related non-relapse mortality. Bone Marrow Transplant 52: 1452-1454.

11. Bluestone H, Kronman MP, Suskind DL (2017) Fecal microbiota transplantation for recurrent Clostridium difficile infections in pediatric hematopoietic stem cell transplant recipients. J Pediatric Infect Dis Soc.

12. Van Nood E, Speelman P, Kuijper EJ, Keller JJ (2009) Struggling with recurrent Clostridium difficile infections: is donor faeces the solution? Euro Surveill 14.

13. Goloshchapov OV, Kucher MA, Suvorova MA, Klementeva RV, Scherbakov AA, et al. (2017) A first experience of therapy of multi-resistant infectious complications associated with Clostridium difficile and Klebsiella pneumoniae, using a method of fecal microbiota transplantation in patients after allogeneic hemopoietic stem cell transplantation. Infekc. Bolezni 15: 65-74.

14. Kakihana K (2016) Fecal microbiota transplantation for patients with steroid-resistant acute graft-versus-host disease of the gut. Blood 128: 2083-2088. 